\title{
Light resonances from fundamental composite Higgs models: LHC and future colliders
}

\section{Cacciapaglia Giacomo*广}

Université de Lyon, F-69622 Lyon, France: Université Lyon 1, Villeurbanne CNRS/IN2P3,

UMR5822, Institut de Physique Nucléaire de Lyon.

E-mail: g.cacciapaglia@ipnl.in2p3.fr

Light composite states naturally arise in models of Composite Higgs. In particular, in models featuring an underlying gauge-fermion description, the properties of those companions of the composite Higgs can be predicted. We focus on a special fellow, ubiquitous to all models, associated to an anomaly-free U(1). Various searches for resonances already give the most stringent bounds on the Higgs decay constant. We also propose a boosted ditau search to cover the elusive mass range $14<M_{A}<65 \mathrm{GeV}$.

The 39th International Conference on High Energy Physics (ICHEP2018)

4-11 July, 2018

Seoul, Korea

* Speaker.

${ }^{\dagger}$ I acknowledge the contribution of T.Flacke, G.Ferretti and H.Serôdio. 


\section{Introduction}

Models where the Higgs boson arises as a pseudo-Nambu-Goldstone boson (pNGB) of a spontaneously broken global symmetry are a valid alternative to the Standard Model (SM) Higgs sector, as they naturally explain the lightness of the Higgs boson at $125 \mathrm{GeV}$ compared to other new physics states. Once this idea is implemented in composite models [1], the large hierarchy with the Planck scale is also addressed by removing any fundamental scalar in the theory. While the idea that the electroweak symmetry breaking may be due to a strong dynamics is as old as the SM itself [2], it has received a decisive revival in the last decade. The new key ingredient are the presence ofnear conformal dynamics above the condensation scale [3], and the implementation of partial compositeness for the SM fermions [4].

In this work we will follow a more traditional approach to strong dynamics, based on the definition of underlying gauge-fermion interactions. The most attractive possibility is to separate the underlying fermions that generate the Higgs as a pNGB, $\psi$, from the ones that carry QCD colour [5], $\chi$. The latter are necessary to generate partial compositeness for the top quark, as they give QCD colour to the spin-1/2 bound states that mix with the top. What distinguishes the two species is their representation under the confining gauge group. Tight limits apply to the models: the number and representation of fermions is limited by requiring confinement at low energy, while a minimal number is necessary to have a composite Higgs in the spectrum together with colour triplet fermionic bound states. As a result, we are left with only 12 models [6, 7], whose properties are listed in Table 1.

As the allowed Higgs cosets are always non minimal, the spectrum will always contain light electroweakly charged states, together with the ones carrying QCD charges deriving from the confinement of the $\chi$ fermions [7]. Another ubiquitous light state is associated to a spontaneously broken $\mathrm{U}(1)$ symmetry acting on both species of underlying fermions. The charges are defined by the vanishing of the anomaly with the confining gauge symmetry, and they are defined in column 5 of the Table. The latter will be the main focus of this proceedings. Those remarkable pseudo-scalars are potentially very light, with masses down to few tens of $\mathrm{GeV}$, and have coupling to gauge bosons and to the top determined by the topological anomaly and the charges of partial compositeness operators, respectively [7]. Another interesting state is associated to the anomalous $\mathrm{U}(1)$. It will naturally pick up a large mass from the instantons associated to the anomaly, however such contributions may be small. The properties of the two states have been extensively studied in Refs $[7,8]$.

\section{Bounds on light composite resonances}

The light pseudo-scalars can be copiously produced at the LHC via gluon fusion. The fact that their wave function always includes the QCD charged $\chi$ fermions guarantees the presence of such couplings. Another possible production channel is via the decay of top partners [9].

After production, they will dominantly decay into a pair of gauge bosons, or into tops, with rates that are calculable and differ from model to model. We refer the interested reader to Ref. [7] where an extensive study of their coupling and phenomenology is reported. Decays into light fermions, like b's, taus and muons, may also be relevant at lower masses. As the final state is a 


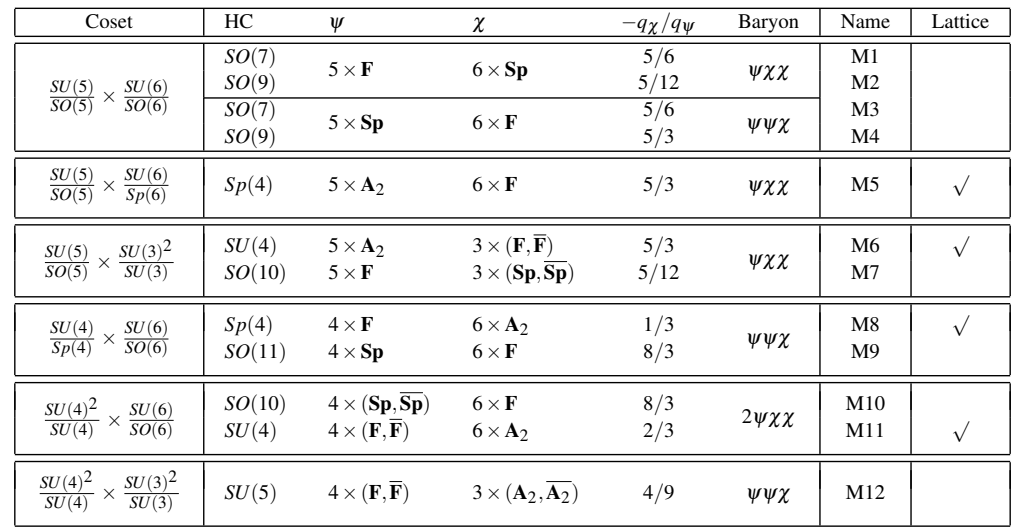

Table 1: Model details showing the cosets, hypercolour gauge group and fermion representations. The nomenclature follows Ref. [7], while the last columns indicate the models that are currently being considered on the lattice. Note that $\mathbf{S p}$ indicates the spinorial representation of $S O(N)$ while $\mathbf{F}$ and $\mathbf{A}_{2}$ the fundamental and two-index anti-symmetric respectively.

simple resonance, for each model we can extract a bound on the production rate for the light states. In the following we will denote by $a$ the light state and $\eta^{\prime}$ the heavy one, which roughly correspond to the anomaly free and anomalous U(1) respectively. In turn, the bound on the cross section can be expressed in terms of a lower limit on the decay constant $f$ of the Higgs. In Fig. 1 we show the lower bounds on $f$ as a function of the masses of the two states. We combine various searches, indicated in the legenda, by taking the limit from the strongest one. The plots refer to model M6 (see Table 1). Note that the typical lower bound on $f$ coming from electroweak precision tests is $f \gtrsim 1 \mathrm{TeV}$. We see, therefore, that in a large portion of the parameter space, the constraint from the non-discovery of the light pseudo-scalars is already much stronger. However, the plot shows a low sensitivity area for heavy $\eta^{\prime}$ and $a$ between $14<M_{a}<65 \mathrm{GeV}$. This mass range lies above a specific low mass di-muon from CMS, and the low mass di-photon searches. The strongest bound here comes from the indirect constraint on decays of the Higgs in new physics, which will not improve much in the future.
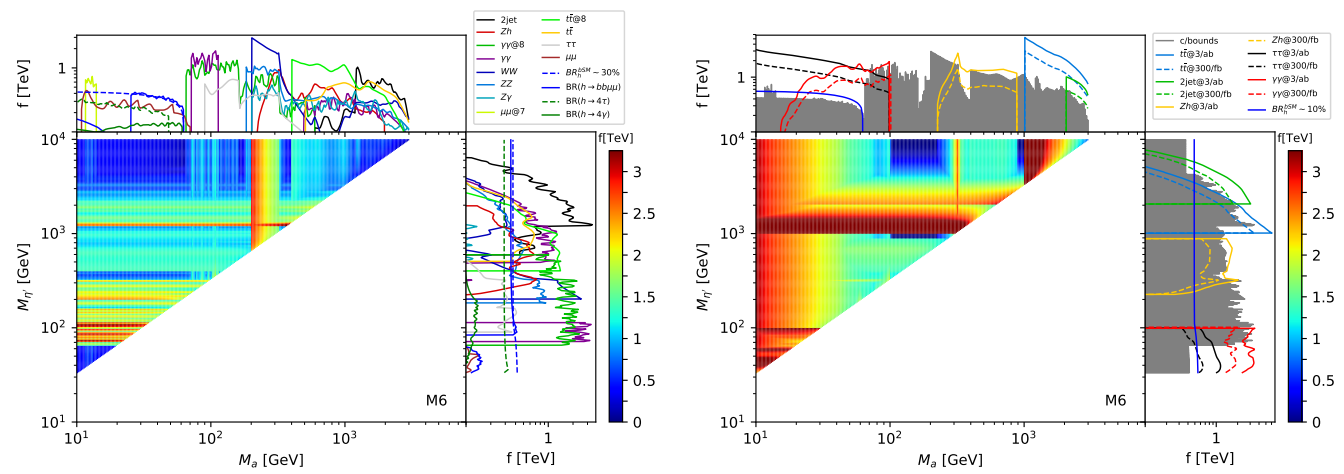

Figure 1: Lower limit on $f$ for model M6 in the $a-\eta^{\prime}$ mass plane. On the left the current bounds, on the right High-Luminosity projections. 
To close this gap, in Ref. [8] we proposed to search for a boosted pair of taus, which come from the decay of $a$ after recoiling against a jet. We simulated the signal and background for decays of the taus in two different flavour leptons. The main discriminant against the backgrounds is the small separation angle between the leptons. This, however, would require a dedicated search at the LHC experiments that lessens the isolation criterium on leptons. In Fig. 2 we show the projected sensitivity for various choices for a minimal cut on the separation angle between the leptons, showing the gain at low invariant mass. The High-Luminosity run of the LHC would, therefore, push the bound on $f$ above the TeV for M6. For the other shown model, M8, the ditau search would be less sensitive. A complementary bound can be extracted from the measurement of the photon spectra at the LHC, as suggested in Ref. [10].

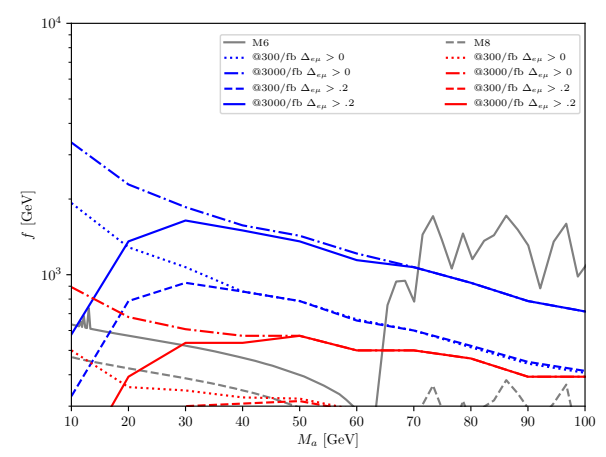

Figure 2: Projected limit on $f$ from the boosted ditau search. Shown in grey are the current bounds as of Figure 1.

In the right panel of Fig. 1 we show the High-Luminosity projections of various searches compared to the current bounds (in shaded grey). We see how the complementarity between the ditau channel and the photon bound allows to close the low mass region.

\section{References}

[1] D. B. Kaplan and H. Georgi, Phys. Lett. 136B (1984) 183.

[2] S. Weinberg, Phys. Rev. D 13 (1976) 974 Addendum: [Phys. Rev. D 19 (1979) 1277].

[3] B. Holdom, Phys. Rev. D 24 (1981) 1441.

[4] D. B. Kaplan, Nucl. Phys. B 365 (1991) 259.

[5] G. Ferretti and D. Karateev, JHEP 1403 (2014) 077 [arXiv:1312.5330 [hep-ph]].

[6] G. Ferretti, JHEP 1606 (2016) 107 [arXiv:1604.06467 [hep-ph]].

[7] A. Belyaev, G. Cacciapaglia, H. Cai, G. Ferretti, T. Flacke, A. Parolini and H. Serodio, JHEP 1701 (2017) 094 Erratum: [JHEP 1712 (2017) 088] [arXiv:1610.06591 [hep-ph]].

[8] G. Cacciapaglia, G. Ferretti, T. Flacke and H. Serodio, Eur. Phys. J. C 78 (2018) no.9, 724 [arXiv:1710.11142 [hep-ph]].

[9] N. Bizot, G. Cacciapaglia and T. Flacke, JHEP 1806 (2018) 065 [arXiv:1803.00021 [hep-ph]].

[10] A. Mariotti, D. Redigolo, F. Sala and K. Tobioka, Phys. Lett. B 783 (2018) 13 [arXiv:1710.01743 [hep-ph]]. 